\title{
The effect of Epigallocatechin-3-Gallate in allergic airway inflammation*
}

\author{
Yoon Seok Choi' ${ }^{1}$, Chang Hoon Bae', Si-Youn Song ${ }^{1}$, Yong-Dae Kim¹,2 \\ ' Department of Otorhinolaryngology - Head and Neck surgery, College of Medicine, Yeungnam University, Daegu, \\ Republic of Korea \\ ${ }^{2}$ Regional Center for Respiratory Diseases, Yeungnam University Medical Center, Daegu, Republic of Korea
}

Rhinology 52: 0-0, 2014

DOl:10.4193/Rhino13.189

*Received for publication:

October 28, 2013

Accepted: May 23, 2014

\begin{abstract}
Background: The variety of beneficial effects of green tea has been reported. Epigallocatechin-3-gallate (EGCG) is a major component of green tea extract. The biological activity of EGCG includes anti-allergic and anti-inflammatory activities. However, the precise effect of EGCG on the allergic airway inflammation has not been fully defined.
\end{abstract}

Methodology: In lung tissues of an asthma model-mouse, and nasal epithelial cells of patients with allergic rhinitis, the effect and brief signaling pathway of EGCG on MUC5B expression were investigated using real-time polymerase chain reaction, immunohistochemical, and immunoblot analysis.

Results: In the asthma model-mouse, mucus production, MUC5B expression, p38 mitogen-activated protein kinase (MAPK) and matrix metalloprotease (MMP)-9 expression of the asthma group were significantly higher than in the control group. Extracellular signal related kinase (ERK)1/2 MAPK expression did not change. Mucus production, MUC5B expression, p38 MAPK expression, and MMP-9 expression of the asthma + EGCG group were significantly lower than in the asthma group. In the nasal epithelial cells of patients with allergic rhinitis, EGCG significantly decreased phorbol 12-myristate 13-acetate (PMA)-induced MUC5B and MMP-9 expression.

Conclusion: These results suggest that EGCG reduces mucin expression in the asthma model-mouse and nasal epithelial cells of patients with allergic inflammation.

Key words: Epigallocatechin-3-gallate, MUC5B, asthma, allergic rhinitis, nasal epithelial cell

\section{Introduction}

Overproduction of mucus plays an important pathophysiologic factor in allergic airway inflammations, such as asthma and allergic rhinitis. The excessive mucus at respiratory tracts causes inflammation by bacterial infection. Furthermore, the mucus may become more densely packed and can lead to airway closure ${ }^{(1)}$. About $2 \%$ of mucus is comprised about of mucins. Mucins are highly glycosylated proteins which are responsible for viscoelastic properties of mucus ${ }^{(2)}$. Mucins are subdivided into secretory and membrane associated forms ${ }^{(3)}$. Among the mucins, MUC5AC, MUC5B, and MUC8 are representative secretory mucin genes in the human airway ${ }^{(1)}$. In the allergic airway inflammation, airway mucins are produced and secreted in increased quantities ${ }^{(4)}$.

Epigallocatechin-3-gallate (EGCG), a major component of green tea extract, has various functions including reduction of cholesterol and antioxidant activity, which prevents cellular injury due 
to oxidative stress ${ }^{(5-7)}$. Recently, several studies have reported the anti-inflammatory or anti-allergic effect of EGCG: EGCG can counteract allergic asthma-like reaction in sensitized guinea pigs by modulating nitric oxide synthase activity ${ }^{(8)}$. EGCG increases inflammatory cell migration by suppressing matrix metalloprotease (MMP)-9 production and reactive oxygen species (ROS) generation in the murine model of asthma ${ }^{(9)}$. However, the precise mechanism of action about anti-inflammatory and allergic effects of EGCG has not been totally defined. In our previous study on mucin-producing human $\mathrm{NCl}-\mathrm{H} 292$ airway epithelial cells, the results revealed that EGCG down-regulates phorbol 12-myristate 13-acetate (PMA)-induced MUC5B gene expression: p38 mitogen-activated protein kinase (MAPK) and MMP-9 may be an important mediator of PMA-induced MUC5B expression ${ }^{(10)}$. In the present study, we aim to determine the effect and the brief signaling pathway of EGCG in the asthma model-mouse and the nasal epithelial cells of patients with allergic rhinitis.

\section{Materials and methods}

Materials EGCG, ovalbumin (OVA), and PMA were obtained from SigmaAldrich (St Louis, MO, USA). RPMI 1640 medium and Trizol were obtained from Invitrogen (Carlsbad, CA, USA). Real-time polymerase chain reaction (PCR) kits were obtained from Roche Applied Science (Mannheim, Germany). Fetal bovine serum (FBS) was obtained from Hyclone Laboratories (Logan, UT, USA). Primary antibody and anti-goat or anti-mouse horseradish peroxidase (HRP)-conjugated secondary antibody of MUC5B, monoclonal human MMP-9 antibody, and $\beta$-actin were obtained from Santa Cruz Biotechnology (Santa Cruz, CA, USA). Extracellular signal related kinase 1/2 (ERK1/2) and p38 antibodies were purchased from Cell Signaling Technology (Danvers, MA, USA). Aluminum hydroxide was obtained from Pierce Biotechnology (Rockford, IL, USA).

\section{Asthma model-mouse}

BALB/c mice, 6-weeks-old and weighing $20 \mathrm{~g}$, were purchased from Jung Ang Lab. Animal (Seoul, South Korea). They were housed in the animal unit of College of Medicine at Yeungnam University in a temperature and moisture controlled room under a regulated 12 hours light/dark cycle. Mice were subjected to an adaptation period of one week and were divided into three groups for experiment: normal control, asthma, and asthma + EGCG. The mice were cared and treated in accordance with the guidelines for the care and use of laboratory animals provided by Yeungnam University. This study was approved by the institutional review board at the Yeungnam University Medical Center.

\section{OVA sensitization, challenge, and administration of EGCG} On days 0 and 14, the mice were sensitized with an intraperito- neal injection of $20 \mu \mathrm{g}$ of OVA and $2 \mathrm{mg}$ of aluminum hydroxide dissolved in $0.2 \mathrm{~mL}$ of phosphate buffered saline (PBS). On days 28-30, the mice were challenged through the airway with aerosol of $1 \%$ OVA dissolved in PBS for 1 hour. EGCG (10 mg/kg/day) was administered intraperitoneally once daily on days 21-25. Negative control mice were treated intraperitoneally with PBS once daily on days 21-25. The mice were sacrificed on day 31.

Histologic studies of mucus and immunohistochemical analysis of MUC5B protein

Forty eight hours after the last OVA challenge, the lungs were removed from the mice. The lung tissues were fixed in $10 \%$ formalin, embedded in paraffin, and sectioned to a thickness of 4 $\mu \mathrm{M}$. The sections were stained with the periodic acid Schiff (PAS) reagent to investigate the mucus production.

Immunohistochemical analysis was performed by the avidinbiotin-peroxidase method. Paraffin-embedded $6 \mu \mathrm{M}$ sections were deparaffinized and rehydrated. For immunostaining of MUC5B, primary, monoclonal antibody anti-MUC5B was used followed by the proper secondary antibody. For the negative control, non-immune serum immunoglobulin (lg)G, instead of primary antibody, was used. The slides were counterstained with hematoxylin and mounted. Immunoreactivity for MUC5B was evaluated for the intensity of staining. The results were expressed as a scoring system that was determined by two independent observers on blinded slides, where scores - and + corresponded to the absence and the presence of a brown stain of positive intensity.

\section{Cell culture and treatment}

For the primary culture of human nasal epithelial cells with allergic rhinitis, mucosa of inferior turbinate was obtained from 10 patients with allergic rhinitis who underwent inferior turbinectomy (five men and five women, mean age: 31.6 years). Diagnosis of allergic rhinitis was based on the patient's history, physical examination, allergen skin-prick tests, and specific IgE assay. All patients met the standard criterion of allergic rhinitis issued by the Allergic Rhinitis and Its Impact on Asthma guidelines. All subjects discontinued oral and topical corticosteroid and antibiotic therapy for at least four weeks before surgery. The nasal mucosal tissue was washed with PBS and immersed in dispase (Boehringer Mannheim Biochemica, Mannheim, Germany) for 90 minutes. After the tissue was scraped off the surface of the nasal mucosa with a scalpel, it was added to $1 \%$ PBS and filtered through a mesh. The cells were seeded in a 24-well plate at 2.5 $\times 10^{5}$ cells/well and were then incubated with EpiLife medium and human keratinocyte growth supplement $(5 \mathrm{~mL} / 500 \mathrm{~mL}$ of medium). When the cultures were confluent, the cells were exposed to the indicated concentrations of EGCG for 1 hour after pretreatment with PMA (10 nM) for 8 hours. 
Real-time PCR analysis of MUC5B and MMP-9 mRNA expression

Isolation of total RNA from the cultured cells was performed according to the manufacturer's instructions (Roche Applied Science). Real-time PCR was performed with the LC Fast Start DNA Master SYBR Green kit (Roche Applied Science) using 0.5 $\mu \mathrm{L}$ of $\mathrm{CDNA}$, corresponding to $25 \mathrm{ng}$ of total RNA in a $10 \mu \mathrm{L}$ final volume, $2.5 \mathrm{mM} \mathrm{MgCl} 2$ and $0.5 \mu \mathrm{M}$ of each primer (final concentration). Quantitative PCR was performed using a LightCycler (Roche Applied Science) for 45 cycles at $95^{\circ} \mathrm{C}$ for 10 seconds, specific annealing temperature for 5 seconds and $72^{\circ} \mathrm{C}$ for 10 seconds. Data were normalized to glyceraldehydes-3-phosphate dehydrogenase (GAPDH). Amplification specificity was evaluated using the melting curve following the manufacturer's instructions (Roche Applied Science). Primer sequences and conditions used were according to previously published studies of MUC5B, MMP-9, and GAPDH ${ }^{(6,11)}$.

\section{Immunoassay for MUC5B protein}

MUC5B protein level was determined by an enzyme-linked immunosorbent assay (ELISA). The sample of cell lysates from the extracted lung tissues of asthma model-mouse was incubated at $40^{\circ} \mathrm{C}$ in wells of a 96 -well plate. Plates were blocked with $2 \%$ bovine serum albumin and incubated with primary antibody against MUC5B diluted with PBS containing 0.05\% Tween 20 for 1 hour. The wells were incubated with HRP-conjugated secondary antibody of MUC5B in each well. After 4 hours, color was developed using 3, 3', 5, 5'-tetramethylbenzidine peroxidase solution and stopped with $2 \mathrm{~N}^{-} \mathrm{H}_{2} \mathrm{SO}_{4}$. Optical density measurements were obtained from an ELISA reader (EL800; BIO-TEK Instruments, Winooski, VT, USA) at $450 \mathrm{~nm}$.

\section{Western blot analysis of p38 MAPK, ERK1/2 MAPK, and}

\section{MMP-9}

Samples of the extracted lung tissues from asthma model-mice were seeded in wells of a 6-well plate and treated with EGCG.

The cells were exposed to trypsin, and formed into pellets at 700 $g$ at $4^{\circ} \mathrm{C}$ and pellets were resuspended in lysis buffer. The preparation was then clarified by centrifugation, and the supernatant was saved as a whole-cell lysate. Proteins $(20 \mu \mathrm{g})$ were separated using $10 \%$ reducing Sodium dodecyl sulfate-polyacrylamide gel electrophoresis and electroblotted onto a nitrocellulose membrane. The membrane was blocked with $5 \%$ nonfat dry milk, followed by incubation with the indicated primary antibody against p38, ERK1/2, or human monoclonal MMP-9 antibody for 4 hours. Subsequently, the membrane was incubated for 1 hour with the proper secondary antibody conjugated to HRP, and developed using an enhanced chemiluminescence kit (Perkin Elmer Life Sciences). Bands were detected after exposure to $\mathrm{x}$ ray film for 10 seconds.
A

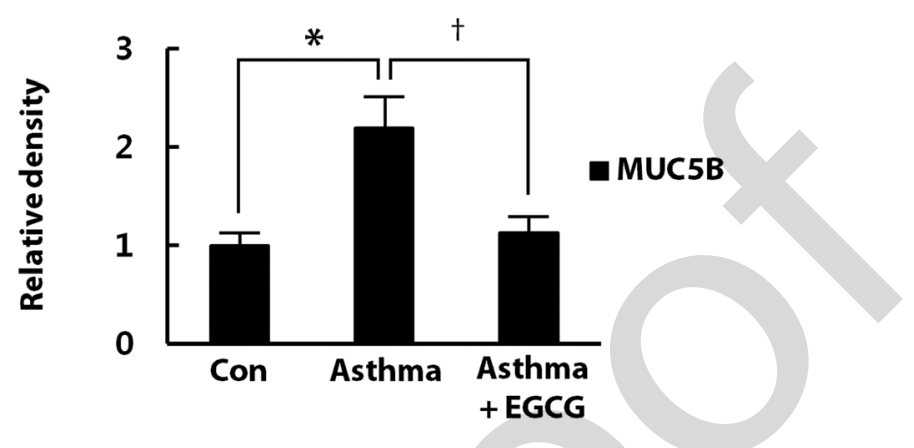

B

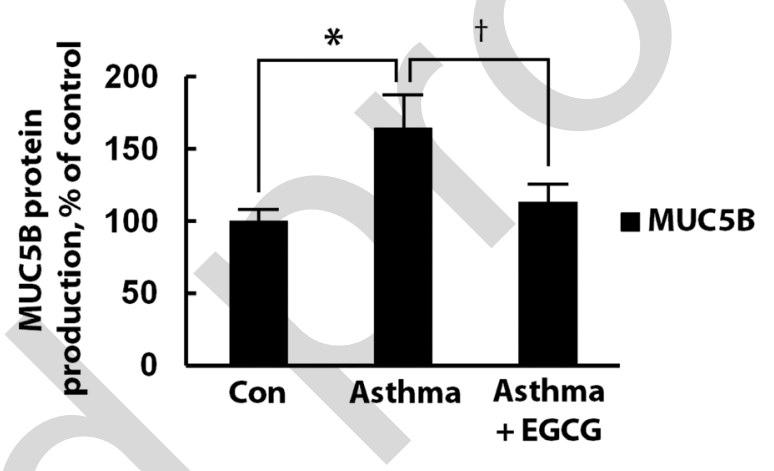

Figure 1. Effect of EGCG on MUC5B mRNA expression and protein production in the asthma model-mouse. Real-time PCR showed that MUC5B mRNA expression of the asthma group was significantly higher than the control group, and MUC5B mRNA expression of the asthma + EGCG group was significantly lower than asthma group; (A). ELISA showed that MUC5B protein production of the asthma group was significantly higher than the control group, and MUC5B protein production of the asthma + EGCG group was significantly lower than the asthma group; (B). Bars represent the averages \pm S.D. of three independent experiments in triplicate. ${ }^{*} p<0.05$ compared with the control, $+p<0.05$ compared with the asthma. Con, control.

\section{Statistical analysis}

Statistical analysis was performed using SPSS, version 12.0 (SPSS, Chicago, IL, USA). Data comparison was done with Kruskal-Wallis ANOVA test, followed by the Mann-Whitney test as a post hoc analysis. For all tests, a $\mathrm{p}$-value $<0.05$ was considered statistically significant.

\section{Results}

EGCG decreases mucus production and MUC5B expression in the asthma model-mouse

To investigate the effect of EGCG on MUC5B expression in the asthma model-mouse, MUC5B mRNA expression and protein production were analyzed by real-time PCR and ELISA. MUC5B mRNA expression and protein production of the asthma group were significantly higher than the control group. MUC5B mRNA expression and protein production of the asthma + EGCG group 

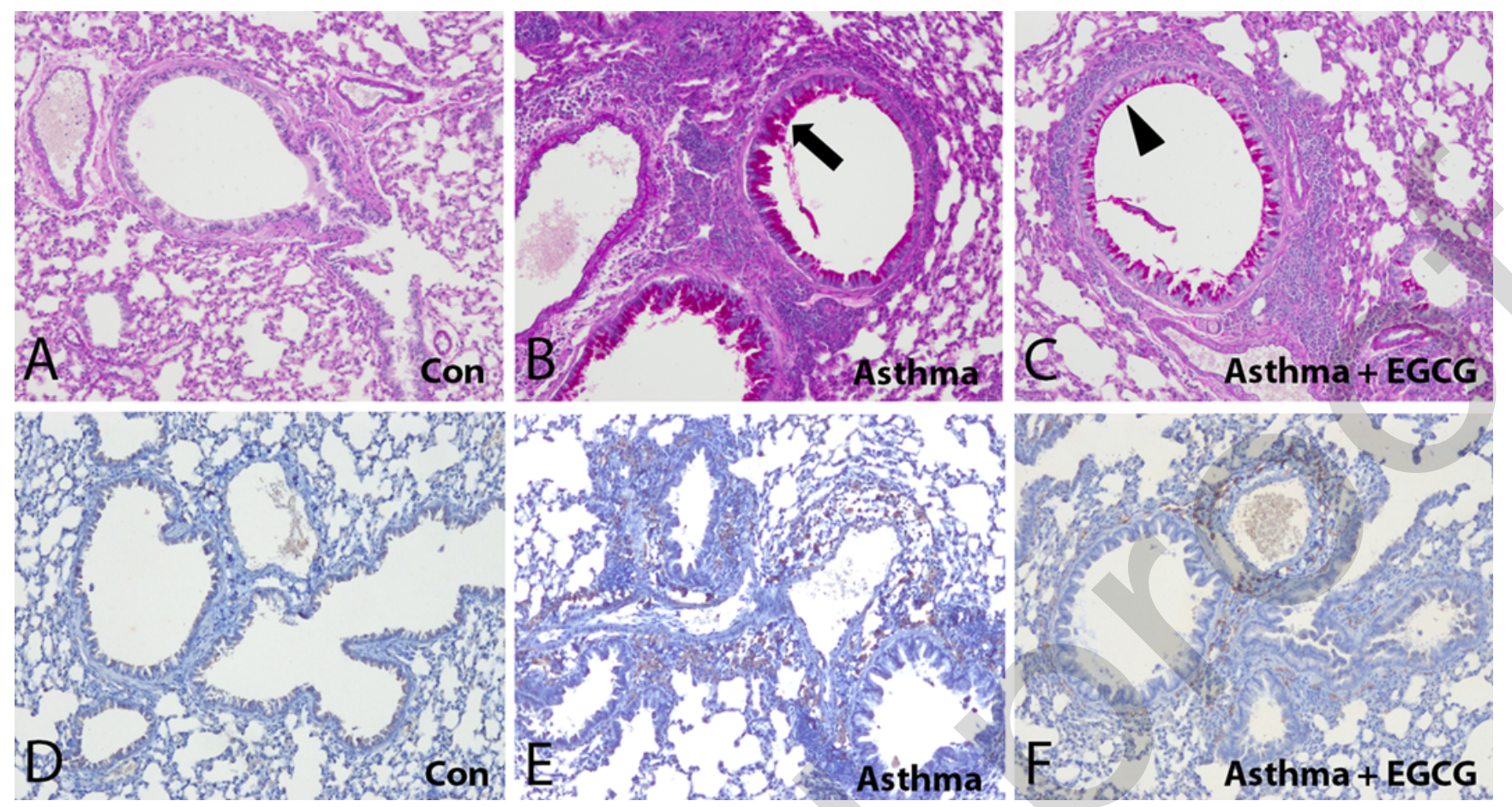

Figure 2. Effect of EGCG on mucus production in histological study of the asthma model-mouse. PAS staining of the bronchiole showed the control group; (A), increased amount of mucus production (arrow) in the asthma group; (B), and decreased mucus production (arrow head) in the asthma + EGCG group; (C) than in the asthma group; (B) (original magnification, $\times 100$ ). Immunohistochemical staining of the bronchiole showed the control group; (D), increased immunoreactivity for MUC5B in the asthma group; (E), and decreased immunoreactivity for MUC5B in the asthma + EGCG group; (F) than in the asthma group; (E): immunopositive cells appeared brown (original magnification, $\times 100$ ).

were significantly lower than the asthma group (Figures $1 \mathrm{~A}$ and B).

EGCG decreases mucus production in histological study of the asthma model-mouse

To confirm the effect of EGCG on mucus production and MUC5B expression in the asthma model-mouse, a histologic study and immunohistochemical analysis were performed. Increased amount of mucus production was observed in the bronchioles of the asthma group (Figure $2 \mathrm{~B}$ ), compared to the control group (Figure 2A), and decreased mucus production in the asthma + EGCG group (Figure $2 \mathrm{C}$ ) than in the asthma group. In addition, increased immunoreactivity for MUC5B was observed in the bronchioles of the asthma group (Figure 2E), compared to the control group (Figure 2D), and decreased immunoreactivity for MUC5B in the asthma + EGCG group (Figure 2F) than in the asthma group.

EGCG inhibits P38 MAPK and MMP-9 expression in the asthma model-mouse

To investigate the brief signaling pathway associated with EGCG in the asthma model-mouse, ERK1/2 and p38 MAPK expression were analyzed by Western blot. p38 MAPK expression of the asthma group was significantly higher than the control group, and p38 MAPK expression of the asthma + EGCG group was significantly lower than the asthma group. However, ERK1/2 MAPK expression did not change (Figure $3 \mathrm{~A}$ ). To investigate the effect of EGCG on MMP-9 expression in asthma model-mice, MMP-9 expression was analyzed by real-time PCR and Western blot. MMP-9 expression was significantly higher in the asthma group than the control group, and MMP-9 expression of the asthma + EGCG group was significantly lower than the asthma group (Figures $3 \mathrm{~B}$ and $\mathrm{C}$ ).

EGCG inhibits MMP-9 and MUC5B expression in the nasal epithelial cells of patients with allergic rhinitis To evaluate the effect of EGCG on MMP-9 and MUC5B expression in the nasal epithelial cells of patients with allergic rhinitis under inflammatory condition, primary cultured cells was stimulated by PMA, MUC5B and MMP-9 mRNA expression was analyzed by real-time PCR. The result showed that PMA-induces MMP-9 and MUC5B over-expression and this was significantly attenuated by EGCG (Figures $4 \mathrm{~A}$ and $\mathrm{B}$ ).

\section{Discussion}

Allergic airway inflammations, such as asthma and allergic rhinitis, are characterized by airway hyper-responsiveness and mucus overproduction. The properties of mucus depend on its 
A

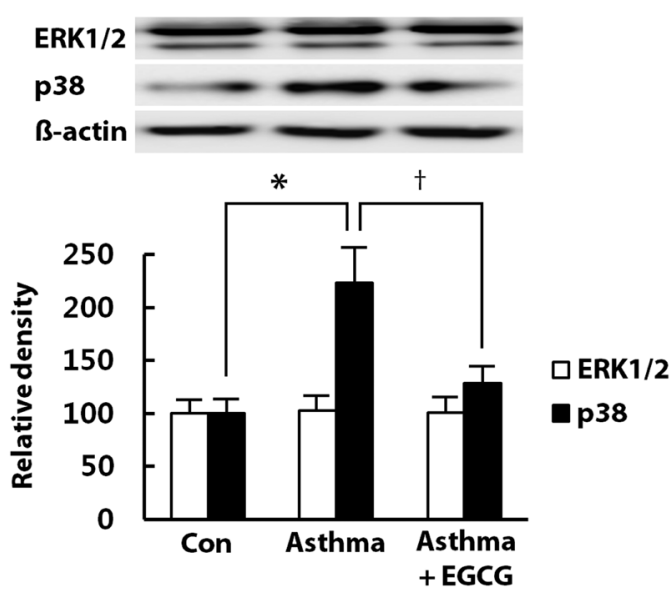

B

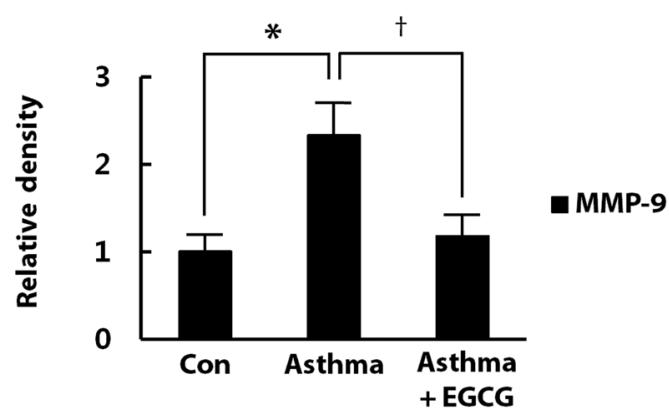

C

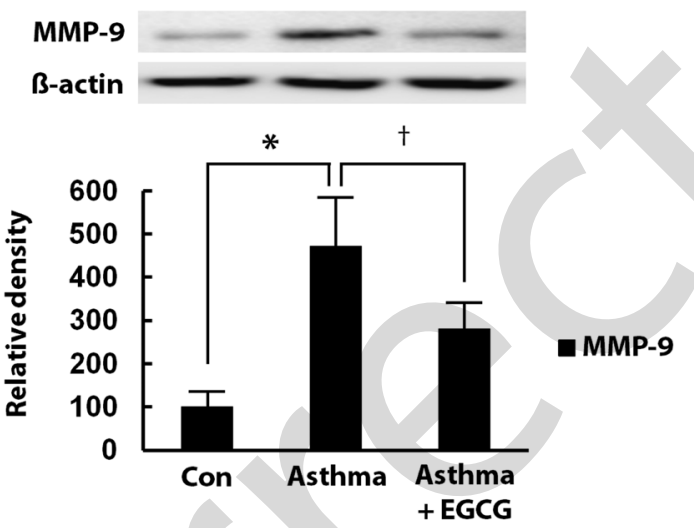

Figure 3. Effect of EGCG on p38 MAPK expression and MMP-9 expression in the asthma model-mouse. Western blot showed that p38 MAPK expression of the asthma group was significantly higher than the control group, and p38 MAPK expression of the asthma + EGCG group was significantly lower than the asthma group. But, ERK1/2 MAPK expression did not change; (A). Real-time PCR showed that MMP-9 mRNA expression of the asthma group was significantly higher than the control group, and MMP-9 mRNA expression of the asthma + EGCG group was significantly lower than the asthma group; (B). Western blot showed that MMP-9 expression of the asthma group was significantly higher than the control group, and MMP-9 expression of the asthma + EGCG group was significantly lower than the asthma group; (C). Bars represent the averages \pm S.D. of three independent experiments in triplicate. ${ }^{*} P<0.05$ compared with the control, $+\mathrm{P}<0.05$ compared with the asthma. Con, control.
A

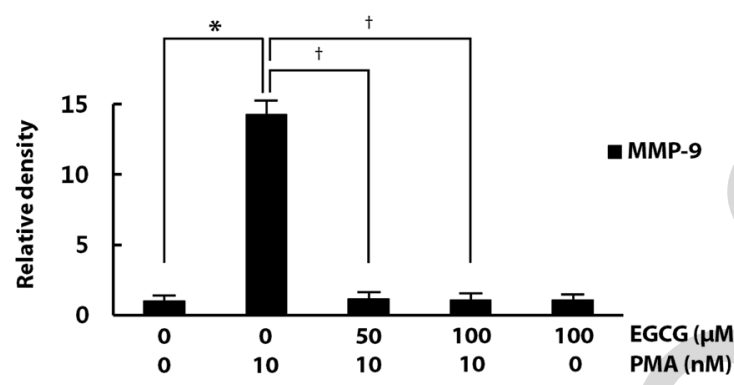

B

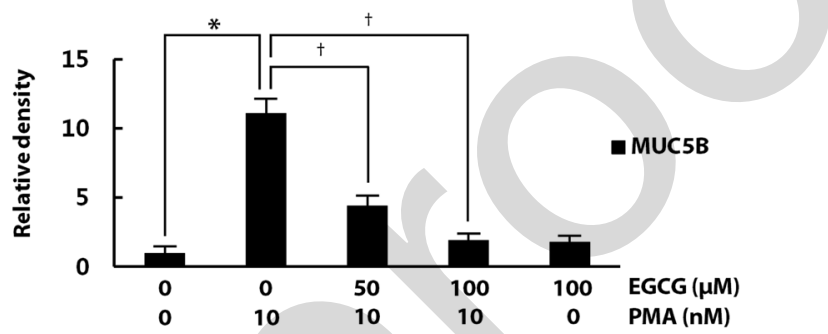

Figure 4. Effect of EGCG on PMA-induced MMP-9 mRNA expression in human nasal epithelial cells of patients with allergic rhinitis. Real-time PCR showed that PMA-induced MMP-9 mRNA expression was significantly decreased by pretreatment with EGCG; (A). PMA-induced MUC5B expression was significantly decreased by pretreatment with EGCG; (B). Bars represent the averages \pm S.D. of three independent experiments in triplicate. ${ }^{*} P<0.05$ compared with zero value, $+P<0.05$ compared with PMA only.

content with mucins, which are regulated by a variety of inflammatory mediators. PMA, a protein kinase C (PKC) activator, has been known as an inflammatory mediator to modulate various cellular events. PMA also plays a significant role in the induction of mucin production in $\mathrm{NCl}-\mathrm{H} 292$ cells through $\mathrm{p} 38$ dependent MMP-9 signaling pathway $\left.{ }^{(10,12,13}\right)$. PMA-induced MUC5B expression is regulated through epidermal growth factor receptor (EGFR) and p38-mediated transcriptional mechanism (13-16). Therefore, to evaluate the effect of EGCG on allergic rhinitis under inflammatory condition, PMA was used as an inflammatory stimulator for influencing primary cultured human nasal epithelial cells of patients with allergic rhinitis.

MMP-9 is a kind of collagenase that cleaves type IV collagen, which makes up a substantial portion of the basement membrane of airways. MMP-9 plays a role in tissue remodeling in diseases such as asthma and bronchiectasis; increased MMP-9 activity in bronchoalveolar lavage fluid was observed in asthmatic patients compared to healthy subjects ${ }^{(17)}$. Several studies have demonstrated that MMP-9 mediates mucin gene expression in airway epithelial cells. Acrolein-activated MMP-9, leading to MUC5AC accumulation, contributes to persistent mucin production in chronic obstructive pulmonary disease ${ }^{(18)}$. Lipopolysacchari- 
de-induced MUC5AC production may be partially mediated by MMP-9 activation ${ }^{(19)}$. The results of our previous study revealed that $\mathrm{P} 38$ MAPK plays a role in the signaling pathway of PMA-induced MUC5B expression, and EGCG can down-regulate MUC5B and MMP-9 expression in human $\mathrm{NCl}-\mathrm{H} 292$ cells. Moreover, MMP-9 may be an important mediator of PMA-induced MUC5B expression, and p38 MAPK plays a role in PMA-induced MMP-9 expression in human $\mathrm{NCl}-\mathrm{H} 292$ airway epithelial cells ${ }^{(10)}$.

EGCG, derived from green tea, is the most abundant bioactive polyphenolic substance. Based on a previous safety and pharmacokinetic study, oral administration of EGCG at a daily dose of $800 \mathrm{mg}$ is safe and well tolerated in healthy human subjects. It is likely that a daily $800 \mathrm{mg}$ bolus of EGCG in humans will approximately achieve levels similar to those in sera of EGCG $(10 \mathrm{mg} / \mathrm{kg} /$ day) intraperitoneally injected mice ${ }^{(20)}$. Therefore, many animal studies related to EGCG, including our own, were performed in the concentration range of $3-20 \mathrm{mg} / \mathrm{kg} /$ day, which is likely to be achieved under physiologic condition and is not expected to cause any negative side effects ${ }^{(8,9,21,22)}$. Previous in vitro studies of EGCG on airway epithelial cell, the concentration range of 10-1000 $\mu \mathrm{M}$ was used, because topical application of EGCG on nasal mucosa might achieve the effective experimental dosage of EGCG, allowing the use of EGCG as a therapeutic agent ${ }^{(6,10,23)}$. Thus, our study using the allergic nasal epithelial cell was performed with EGCG concentrations of 50 and $100 \mu \mathrm{M}$.

From the perspective of the protective effect of EGCG on asthma or asthma-like reaction, EGCG can sustain endothelial nitric oxide synthase (NOS) activity and maintain endogenous nitric oxide (NO) generation at physiological levels in an OVAsensitized asthma model. This can counteract the activation of inducible NOS, which releases excess NO and subsequently activates cyclooxygenase-2 to produce inflammatory prostaglandins ${ }^{(8)}$. In a toluene diisocyanate-sensitized asthma model, EGCG reduced the pathologic lung damage due to the suppression of ROS ${ }^{(9)}$. In an OVA-sensitized asthma model, EGCG reduced inflammatory cell recruitment and overall inflammatory response in airways and lung tissues ${ }^{(22)}$. But there has not been any study on the influence of EGCG on mucin gene expression and mucin production in allergic airway inflammations. There- fore, the present study was performed to assess the effect of EGCG on mucin expression in the allergic airway inflammations. The results showed that increased amount of mucus production in asthma group than the control group, and mucus production was decreased in the asthma + EGCG group. MUC5B, MMP-9, and p38 MAPK expressions were increased in the asthma group and significantly decreased in the asthma + EGCG group. These results suggest that EGCG down-regulates MUC5B expression in the asthma model-mouse. To evaluate the effect of EGCG on allergic rhinitis under inflammatory condition, primary cultured human nasal epithelial cells of patients with allergic rhinitis were stimulated by PMA to make an intensive inflammatory condition. The results also demonstrate that EGCG attenuates MUC5B and MMP-9 expression. We did not investigate the effect of EGCG on human bronchial epithelial cells of patients with asthma in this study. However, we expect the results of such studies to be similar to the results in the current study. Further studies of the effect of EGCG on allergic airway epithelial cells should be performed.

In conclusion, the results of in vivo and in vitro studies suggest that EGCG down-regulates MUC5B expression through the $\mathrm{p} 38$ MAPK dependent MMP-9 signaling pathway in allergic airway inflammations. Although overall signaling pathways for regulation of the mucin genes by EGCG in allergic airway inflammations require further confirmation, EGCG could be a novel therapeutic agent for controlling mucin production in allergic airway inflammations.

\section{Acknowledgements}

This work was supported by a 2013 Yeungnam University Research Grant.

\section{Authorship contribution}

YSC: writing, study design; CHB: data collection, manuscript revision; SYS: data analysis, data interpretation; YDK: study concept, study design, supervision

\section{Conflicts of Interest}

The authors state that there is no conflict of interest regarding this manuscript.

\section{References}

1. Evans CM, Kim K, Tuvim MJ, Dickey BF Mucus hypersecretion in asthma: causes andeffects. Curr Opin Pulm Med 2009; 15 4-11.

2. Williams OW, Sharafkhaneh A, Kim V, Dickey BF, Evans CM. Airway mucus: From production to secretion. Am J Respir Cell Mol Biol 2006; 34: 527-536.

3. Ali MS, Pearson JP: Upper airway mucin gene expression. a review. Laryngoscope
2007; 117: 932-938.

4. Rose MC, Voynow JA. Respiratory tract mucin genes and mucin glycoproteins in health and disease. Physiol Rev 2006; 86: 245-278.

5. Raederstorff DG, Schlachter MF, Elste V, Weber P. Effect of EGCG on lipid absorption and plasma lipid levels in rats. J Nutr Biochem 2003; 14: 326-332.

6. Oliva J, Bardag-Gorce F, Tillman B, French SW. Protective effect of quercetin, EGCG catechin and betaine against oxidative stress induced by ethanol in vitro. Exp Mol Pathol 2011; 90: 295-299.

7. Lee $H$, Bae JH, Lee SR. Protective effect of green tea polyphenol EGCG against neuronal damage and brain edema after unilateral cerebral ischemia in gerbils. J Neurosci Res 2004; 77: 892-900.

8. Bani D, Giannini L, Ciampa A, Masini $E$, Suzuki $Y$, Menegazzi $M$, et al. Epigallocatechin-3-gallate reduces allergen- 
induced asthma-like reaction in sensitized guinea pigs. J Pharmacol Exp Ther 2006 317: 1002-1011.

9. Kim SH, Park HJ, Lee CM, Choi IW, Moon DO, Roh HJ, et al. Epigallocatechin-3-gallate protects toluene diisocyanate-induced airway inflammation in a murine model of asthma. FEBS Lett 2006; 580: 1883-1890.

10. Song EJ, Bae CH, Kim J-Y, Kim Y-W, Park S-Y, Song S-Y, et al. Effect of epigallocatechin3-gallate on PMA-induced MUC5B expression in human airway epithelial cells. Clin Exp Otorhinolaryngol. 2013; 6: 237-242.

11. Wang Y, Shen Y, Li K, Zhang P, Wang G, Gao $L$, et al. Role of matrix metalloproteinase-9 in lipopolysaccharide-induced mucin production in human airway epithelial cells. Arch Biochem Biophys 2009; 486: 111-118.

12. Yuan-Chen Wu D, Wu R, Reddy SP, Lee YC, Chang MM. Distinctive epidermal growthfactor receptor/extracellular regulated kinase-independent and -dependent signaling pathways in the induction of airway mucin 5B and mucin 5AC expression by phorbol 12-myristate 13-acetate. Am Pathol 2007; 170: 20-32.

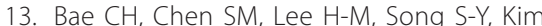
$Y-D$. The Effect of Doxycycline on PMAInduced MUC5B Expression via MMP-9 and p38 in NCl-H292 Cells. Clin Exp Otorhinolaryngol 2011; 4: 177-183.

14. Hewson CA, Edbrooke MR, Johnston SL. PMA induces the MUC5AC respiratory mucin in human bronchial epithelial cells, via PKC, EGF/TGF-alpha, Ras/Raf, MEK, ERK and Sp1-dependent mechanisms. J Mol Biol 2004: 344: 683-695.
15. Park SJ, Kang SY, Kim NS, Kim HM Phosphatidylinositol 3-kinase regulates PMA-induced differentiation and superoxide production in HL-60 cells. Immunopharmacol Immunotoxicol 2002; 24: 211-226.

16. Wu DY, Wu R, Chen Y, Tarasova N, Chang MM. PMA stimulates MUC5B gene expression through an Sp1-based mechanism in airway epithelial cells. Am J Respir Cell Mol Biol 2007; 37: 589-597.

17. Ko FW, Diba C, Roth M, McKay K, Johnson PR, Salome C, et al. A comparison of airway and serum matrix metalloproteinase-9 activity among normal subjects, asthmatic patients, and patients with asthmatic mucus hypersecretion. Chest 2005; 127: 1919-1927.

18. Deshmukh HS, Shaver C, Case LM, Dietsch M, Wesselkamper SC, Hardie WD, et al. Acrolein-activated matrix metalloproteinase 9 contributes to persistent mucin production. Am J Respir Cell Mol Biol 2008; 38 : 446-454.

19. Ren S, Guo LL, Yang J, Liu DS, Wang T, Chen $L$, et al. Doxycycline attenuates acroleininduced mucin production, in part by inhibiting MMP-9. Eur J Pharmacol 2011; 650: 418-423.

20. Chow HH, Cai Y, Hakim IA, Crowell JA, Shahi F, Brooks CA, et al. Pharmacokinetics and safety of green tea polyphenols after multiple-dose administration of epigallocatechin gallate and polyphenon $E$ in healthy individuals. Clin Cancer Res 2003; 9: 3312-3319.

21. Lee YJ, Choi DY, Yun YP, Han SB, Oh KW, Hong JT. Epigallocatechin-3-gallate pre- vents systemic inflammation-induced memory deficiency and amyloidogenesis via its anti-neuroinflammatory properties. J Nutr Biochem 2013; 24: 298-310.

22. Qin S, Alcorn JF, Craigo JK, Tjoeng C, Tarwater PM, Kolls JK, et al. Epigallocatechin-3-Gallate Reduces Airway Inflammation in Mice through Binding to Proinflammatory Chemokines and Inhibiting Inflammatory Cell Recruitment. J Immunol 2011; 186: 3693-3700.

23. Kim HJ, Park SH, Park SY, Moon UY, Lee BD Yoon $\mathrm{SH}$, et al. Epigallocatechin-3-gallate inhibits interleukin-1 $\beta$-induced MUC5AC gene expression and MUC5AC secretion in normal human nasal epithelial cells. J Nutr Biochem 2008; 19: 536-544.

Yong-Dae Kim, MD, PhD Department of Otorhinolaryngology-Head and Neck Surgery, College of Medicine,

Yeungnam University 317-1, Daemyung-5 Dong, Nam-Gu, Daegu, 705-717

Republic of Korea

Tel: +82-53-620-3781

Fax: +82-53-628-7884

E-mail adress:ydkim@med.yu.ac.kr 